\section{Steroids can reduce renal scarring}

Children with febrile urinary tract infections are at risk of developing acute pyelonephritis. Resultant damage to the kidneys can have long-term detrimental consequences, such as hypertension and end-stage renal failure. Although affected patients are commonly treated with antibiotics, there is an inflammatory component to the condition that is often ignored. A team from Taiwan evaluated addition of the glucocorticoid methylprednisolone (MPD) to antibiotic treatment, reporting the promising results of their double-blind prospective study in Pediatrics.

Yuan-Yow Chiou and his team have previously demonstrated that inflammation, rather than bacterial infection, is responsible for renal scar formation in acute pyelonephritis. "Patients with a fever for more than 3 days before receiving treatment and $\mathrm{C}$-reactive protein levels greater than $90.8 \mathrm{mg} / \mathrm{l}$ were significantly more likely to have renal scarring," says Chiou. "No practical clinical methods exist to alleviate the inflammatory response and thus decrease the incidence of renal scars." The investigators noted that synthetic steroid dexamethasone has proven beneficial for infants and children with bacterial meningitis by reducing inflammation and improving neurological complications, and extended this logic to the treatment of acute pyelonephritis.

The study cohort consisted of children (aged between 1 week and 16 years) considered to be at high risk of renal scar formation, based on abnormal renal ultrasonography or an inflammatory volume of $\geq 4.6 \mathrm{ml}$ on technetium99m-labeled dimercaptosuccinic acid (DMSA) scan. Patients were randomly assigned to receive oral MPD (total daily dosage of $1.6 \mathrm{mg} / \mathrm{kg}$ administered in divided doses every $6 \mathrm{~h}$ ) or placebo for 3 days. All children received empirical parenteral antibiotic treatment comprising cephalothin (100 mg/kg per day; every $6 \mathrm{~h}$ ) and gentamicin $(5 \mathrm{mg} / \mathrm{kg}$ per day delivered over a period of 30 minutes every $12 \mathrm{~h}$ ) for a minimum of 3 days.

Follow-up DMSA scan, performed a minimum of 6 months after discharge from hospital, revealed significant improvements in both number and extent of scars for patients in the MPD group $(n=18)$ compared to those who received

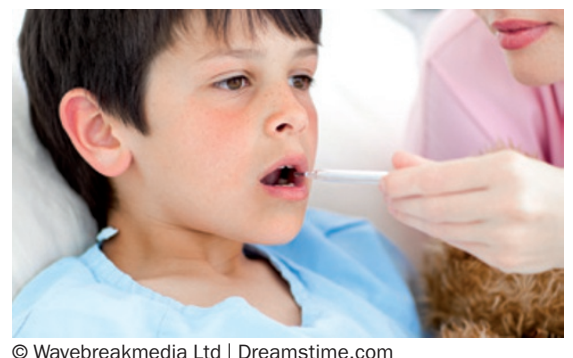

placebo $(n=65)$. Only $33.3 \%$ of children treated with MPD were affected by renal scarring compared to $60 \%$ of those in the placebo group $(P<0.05)$. Median cortical defect volumes were $0 \mathrm{ml}$ (range $0-4.5 \mathrm{ml}$ ) and $1.5 \mathrm{ml}$ (range $0-14.8 \mathrm{ml}$ ) in the MPD and placebo groups, respectively $(P<0.01)$.

Although Chiou is careful to point out that this was a pilot study designed to test their hypothesis, the results are certainly promising. Questions remain regarding the optimal drug dosage, treatment duration and steroid type, and further investigation will hopefully result in a well-designed, large-scale study of adjunctive oral MPD therapy in children with acute pyelonephritis.

Sarah Payton

Original article Huang, Y. Y. et al. Adjunctive oral methylprednisolone in pediatric acute pyelonephritis alleviates renal scarring. Pediatrics 128, e496-e504 (2011) 